\title{
Detección de obstáculos durante vuelo autónomo de drones utilizando SLAM monocular
}

\author{
José Martínez-Carranza ${ }^{1}$, Luis Valentín ${ }^{1}$, Francisco Márquez-Aquino ${ }^{1}$, \\ Juan Carlos González-Islas ${ }^{1}$, Nils Loewen ${ }^{2}$ \\ 1 Instituto Nacional de Astrofísica Óptica y Electrónica, Puebla, \\ México \\ 2 Instituto Tecnológico y de Estudios Superiores de Monterrey, \\ Campus Queretaro, Queretaro, \\ México
}

carranza@inaoep.mx,luismvc@ccc.inaoep.mx, A00889524@itesm.mx

\begin{abstract}
Resumen. En este artículo se describe una metodología para la detección de obstáculos que aparecen de manera repentina durante el vuelo autónomo de un dron. Para lograr lo anterior, este trabajo se basa en el uso de un sistema de localización y mapeo simultaneo o bien, Simultaneous Localisation and Mapping (SLAM) en inglés, monocular. El sistema de SLAM monocular es utilizado para obtener estimaciones de la posición del dron así como un mapa conformado por puntos 3D que representan el ambiente que se observa a través de la cámara a bordo del vehículo. Es importante resaltar que ningún otro sensor de profundidad, de medición de distancia o inercial es utilizado en este trabajo, y que dicho sistema puede ser utilizado de manera indistinta cuando el dron vuela en espacios interiores o exteriores, puesto que la localización del dron y el mapa del ambiente no dependen de la señal GPS o de algún sistema de localización externo, sino únicamente del procesamiento de las imágenes capturadas por la cámara.
\end{abstract}

Palabras clave: Vuelo autónomo, detección de obstáculos, drones, SLAM monocular.

\section{Obstacle Detection during Autonomous Flight of Drones Using Monocular SLAM}

\begin{abstract}
We describe a methodology for the detection of obstacles suddenly appearing during the autonomous flight of a drone. For this, our approach is based on the use of a monocular Simultaneous Localisation and Mapping (SLAM) system. The monocular SLAM system is used to obtain drone's position estimates, as much as to generate a 3D map of the scene, which is observed with an on-board camera. It is important
\end{abstract}


to highlight that no other sensor is used in this work, and that our approach works in both, indoor and outdoor scenes, due to the fact that the drone's localisation does not depend upon GPS signal or any other external localisation system, but only on the processing of the imagery captured by the on-board camera.

Keywords: Autonomous flight, obstacle detection, drones, monocular SLAM.

\section{Introducción}

En los últimos años, la robótica ha sido utilizada con gran interés en diferentes ámbitos, incluyendo aquellos en los que se desarrollan aplicaciones civiles. Algunas organizaciones como DARPA (del inglés, Defense Advanced Research Projects Agency) y RoboCup han impulsado el desarrollo de tecnología para robots móviles autónomos, como lo son humanoides, robots de servicio y vehículos aéreos no tripulados (VANTs) [16, también conocidos como drones.

Recientemente, el uso de drones ha tenido un crecimiento exponencial en la investigación y en el ámbito comercial con un número creciente de aplicaciones, por ejemplo: búsqueda y rescate, vigilancia, inspección aérea de estructuras, monitoreo agroindustrial y forestal, videografía, entre otras. Dichas actividades se realizan tanto en ambientes exteriores como interiores densamente poblados y con un alto dinamismo en el dominio espacio-temporal. Por lo que se requiere que un dron vuele eficientemente de manera autónoma con las capacidad de detección y evasión de obstáculos [3. En ese sentido, la información obtenida por los sistemas de visión a bordo, mejora significativamente las capacidades del dron para realizar dichas tareas. El uso de cámaras como único sensor extereoceptivo en los drones y en los robots en general, provee de información fotométrica detallada que se utiliza por diferentes algoritmos, los cuales determinan la inteligencia del robot para realizar tareas de localización, navegación, seguimiento y manipulación con mayor eficiencia y robustez 18 .

El vuelo autónomo es una capacidad deseada en los drones por diversas razones. Discutiblemente, quizás la más importante es que el dron pueda tomar control de su propio vuelo en caso de que el piloto pierda comunicación con el vehículo, en cuyo caso sería deseable que el dron tuviera la capacidad de identificar dicho evento y por lo tanto volar de manera autónoma hacia algún punto de aterrizaje fuera de riesgo. Actualmente, la solución para lograr un vuelo autónomo es a través de un GPS, el cual trabaja muy bien en exteriores y en áreas libres de obstáculos. Sin embargo, este tipo de sistemas son muy propensos a fallar en ambientes de interiore, dinámicos y densamente poblados. Por lo que, aplicaciones de vigilancia en este tipo de ambientes no podrían ser realizadas con esta solución [10]. Por otra parte, aún cuando el vuelo autónomo se ejecutara con soporte GPS, ésto no libra al vehículo del riesgo de colisionar con objetos que se atraviesen en medio de la ruta de vuelo, por ejemplo: aves, cables, antenas o incluso personas (cuando el vehículo se acerque a tierra). 
Motivados por lo anterior, en este trabajo se propone una metodología para realizar navegación autónoma de un dron con la capacidad de detección de obstáculos. Para esto, se utiliza el sistema SLAM monocular propuesto en [12, para estimar la posición del vehículo; y simultáneamente obtener un mapa de puntos en $\mathbb{R}^{3}$ que representan su entorno. La detección del obstáculo se hace mediante el análisis de las imágenes capturadas con la cámara a bordo. El algoritmo para corregir la posición y controlar la velocidad del dron durante la navegación y detección de obstáculos está basado en un controlador Proporcional Integral Derivativo (PID). Es importante resaltar que ningún otro sensor (por ejemplo, inercial o de profundidad), es utilizado para detectar el obstáculo. Para la evaluación de la metodología propuesta, se utiliza un sistema óptico de captura de movimiento en 3D (Viconß), con el cual se obtiene la posición del dron. Dicho sistema se usa para medir con precisión la distancia a la cuál el algoritmo de control frena y aleja al dron cuando el obstáculo es detectado. Los resultados obtenidos indican que la propuesta aquí presentada es factible y eficaz.

Con el objetivo de describir este trabajo, este artículo ha sido organizado como se indica a continuación: la Sección 2 presenta el trabajo relacionado; la Sección 3 describe la metodología propuesta; los experimentos y resultados se discuten en la Sección 4; las conclusiones y trabajo futuro se presentan en la Sección 5.

\section{Trabajo relacionado}

Una de las tareas principales cuando se navega de manera autónoma con un dron es la detección y evasión de obstáculos. Este tema ha sido investigado ampliamente en aplicaciones para sistemas automáticos de control de tráfico aéreo, en donde se requiere que el vehículo identifique obstáculos en su trayectoria y haga una replaneación de la ruta 1. En ese sentido, la detección de obstáculos, mediante el uso de un telémetro láser, y la replaneación dinámica en helicópteros no tripulados, se han estudiado por Scherer et al. 14 and Shim et al. [15.

El uso de flujo óptico y sensores inerciales ha permitido realizar rutinas de navegación, como son: estimación de distancia [7], evasión de obstáculos [19], [1], estimación de velocidad y altura [5], entre otros. Sin embargo, el flujo óptico acumula error en estimación de trayectorias largas.

El uso de algortímos de visión artificial en los drones ha incrementado de manera sustancial las capacidades del sistema para realizar tareas de navegación. Watanabe [17] aborda el problema de evasión de obstáculos con un helicóptero no tripulado a través de un sistema de visión monocular. Aunque esta propuesta no entrega mediciones absolutas de distancia de los obstáculos, presenta buenos resultados en un ambiente simulado.

Por otra parte, si el movimiento de la cámara es conocido, se puede usar SFM (Del inglés, Structure From Motion) a través del seguimiento de puntos de interés para medir la profundidad de la escena, tal como lo proponen Call et al. [2]. En ese mismo sentido, Hrabar [8] propone el uso de visión estéreo para la 
evasión de obstáculos. Dicha técnica ha sido usada previamente para estimación de movimiento y altura en [13.

\section{Descripción del sistema}

En este trabajo se utiliza un vehículo aéreo comercial de bajo costo de la empresa Parrot, el cual dispone de un SDK (Del inglés, Software Development Kit) para desarrollo. El dron tiene una duración de carga para vuelo de 10 minutos. Además, este dron cuenta con una cámara abordo con la cual se puede capturar video con una resolución de 1920 x 1080 píxeles a 30 cuadros por segundo; el video se transmite de manera inalámbrica (a través de $\mathrm{WiFi}$ ) a la estación terrestre para ser procesada. La Figura 1 muestra una vista en perspectiva del vehículo.

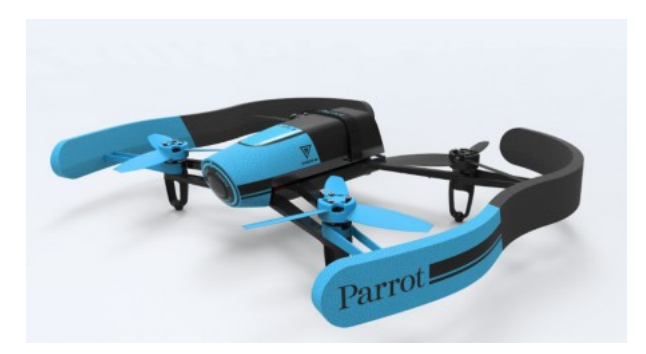

Fig. 1. Vehículo aéreo Bebop. Figura tomada de http://blog.parrot.com

El procesamiento de imágenes y datos para la navegación autónoma y la evasión de obstáculos del dron no se hace a bordo. Para ello se utiliza una computadora portátil con un procesador a 64 bits Intel Core i7-4720HQ a 2.60 GHz x 8, y una memoria RAM de $16 \mathrm{~Gb}$.

El sistema se compone de tres etapas, las cuales son integradas con ROS (Del ingles, Robotic Operative System). La primera etapa consiste en la adquisición de una secuencia de imágenes a través de la cámara del vehículo. La secuencia de imágenes adquiridas es la entrada del algoritmo ORB-SLAM [12] empleado en este trabajo (ver sub-secciń 3.1), el cual permite al sistema móvil localizarse y generar un mapa de puntos en $\mathbb{R}^{3}$ de su entorno, este proceso conforma la segunda etapa del sistema. La tercera etapa es el sistema de control, el cual controla al dron para navegar de manera autónoma y detectar obstáculos. La medición de la distancia a la que el vehículo se detiene para detectar el obstáculo es realizada con el sistema Vicon $®$.

\subsection{ORB-SLAM}

El algoritmo ORB-SLAM es un sistema monocular en tiempo real de localización y mapeo simultáneo basado en la detección de puntos de interés 
representados por descriptores ORB (del inglés, Oriented FAST and Rotated BRIEF). El sistema es robusto a movimientos erráticos, provee las capacidades de relocalización y cierre de trayectoria, así como una inicialización automática de los puntos del ambiente. La Figura 2(a) muestra la imagen de correspondencia del seguimiento de descriptores en el ambiente real observado por la cámara. Mientras que la Figura 2(b) ilustra la representación del mapa de puntos en $\mathbb{R}^{3}$ del ambiente y la localización de la pose de la cámara estimada a través del algoritmo ORB-SLAM. Nótese que en esa figura también se observa el cierre de trayectoria, característica importante del ORB-SLAM.
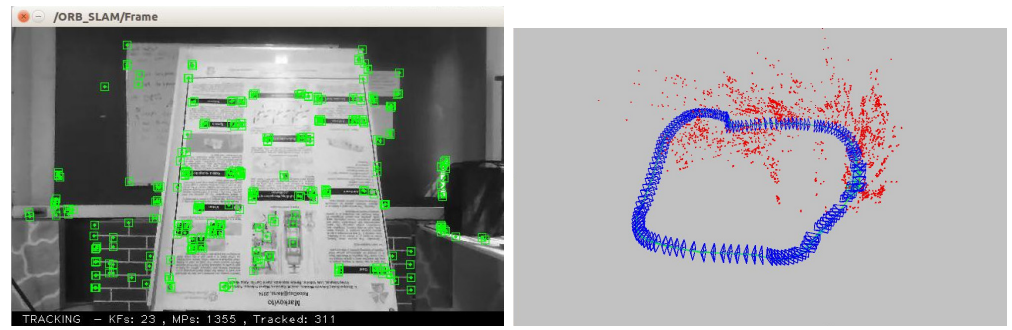

(a) Imagen de correspondencia de (b) Mapa y localización empleando los descriptores en el entorno real ORB-SLAM. Los puntos rojos son empleando ORB-SLAM. los puntos en $\mathbb{R}^{3}$ del ambiente y los cuadros azules representan las poses de la cámara.

Fig. 2. Sistema ORB SLAM en ejecución

El algoritmo ORB-SLAM tiene tres componentes principales, el seguimiento, el mapeo local y el cierre de trayectoria usando descriptores visuales ORB.

La etapa de seguimiento es la encargada de estimar las poses de la cámara mediante la búsqueda de correspondencias de los puntos de interés del mapa entre los frames anteriores y el frame actual. Además, la etapa de seguimiento también toma la decisión de cuando insertar un keyframe para actualizar el grafo basándose en el cambio de información visual entre el keyframe actual y los anteriores. El componente de mapeo local es el que agrega nuevos puntos al mapa y los mantiene. La optimización del mapa construido se hace mediante Bundle Adjustment [6]. Finalmente, la etapa del cierre de trayectoria se emplean cuando un lugar es revisitado. Una vez que el sitio es detectado, se utiliza optimización global para actualizar el mapa. En la Figura 3 se ilustra un diagrama a bloques del sistema ORB-SLAM. Si se requiere información detallada de dicho sistema consulte [12].

\subsection{Sistema de control}

El sistema de control es la etapa encargada de mantener el vuelo autónomo del dron entre dos puntos de referencia. Esto se logra mediante la corrección 
SEGUIMIENTO

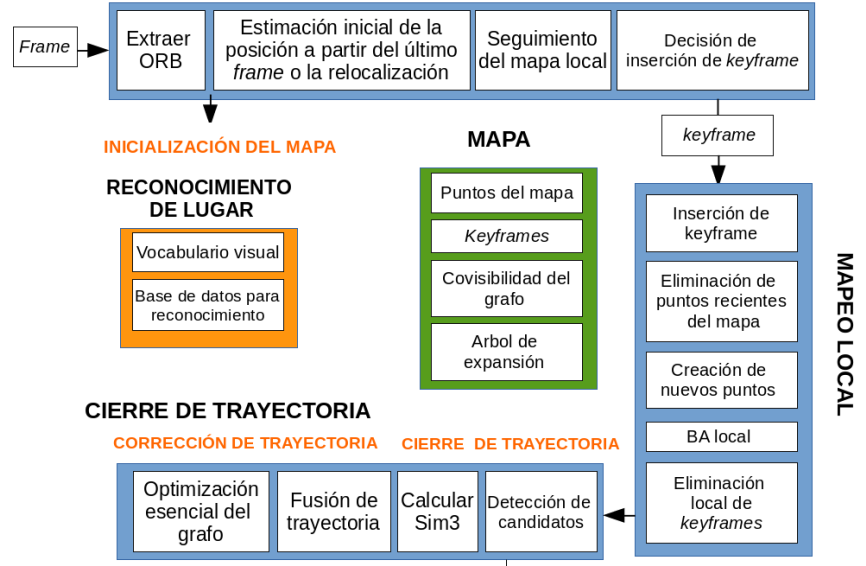

Fig. 3. Panorama general del algoritmo ORB-SLAM. Este diagrama fue adaptado al español de 12 .

del ángulo de elevación ("pitch") y de dirección (ซaw") del vehículo a través de un controlador proporcional integral derivativo (PID). El controlador PID es una de las formas más comunes de control por retroalimentación de un sistema en donde se desea reducir el error entre un valor medido y una referencia. La ecuación de un controlador PID se describe mediante la Ecuación 1.

$$
u(t)=k_{p} e(t)+k_{i} \int_{o}^{t} e(\tau) d \tau+k_{D} \frac{d e(t)}{d t} .
$$

En la Ecuación 1, $u$ es la señal de control, $e=y_{e s p}-y$, es el error de control, $y$ es la variable medida, $y_{e s p}$ es la señal de referencia.

La señal de control es la suma de tres términos, el componente proporcional al error, el componente proporcional a la integral del error y el componente proporcional a la derivada del error, en cada uno de estos términos se definene las constantes $k_{p}, k_{D} \mathrm{y} k_{i}$ respectivamente. Las constantes $k_{p}, k_{D} \mathrm{y} k_{i}$ son determinadas experimentalmente En nuestro caso, la referencia del controlador PID es dinámica y se calcula con la distancia mínima entre el promedio de los puntos del mapa y la posición del vehículo, sobre el eje en el que el dron se desplaza. En este caso se emplea como referencia un umbral de 3/4 de la distancia mínima.

En la Figura 4 se muestra un diagrama de flujo en el que se observa el esquema de control y los bloques que conforman el detector de obstáculos. Puede apreciarse que hay dos controles PID, uno que mantiene al vehículo desplazándose hacia el frente, siempre que no haya algún obstáculo en la trayectoria del vehículo, y otro que lo hace retroceder en caso de que la detección de un obstáculo sea positiva; el vehículo retrocederá hasta que éste regrese al origen de coordenadas para así reiniciar nuevamente el vuelo hacia adelante. 


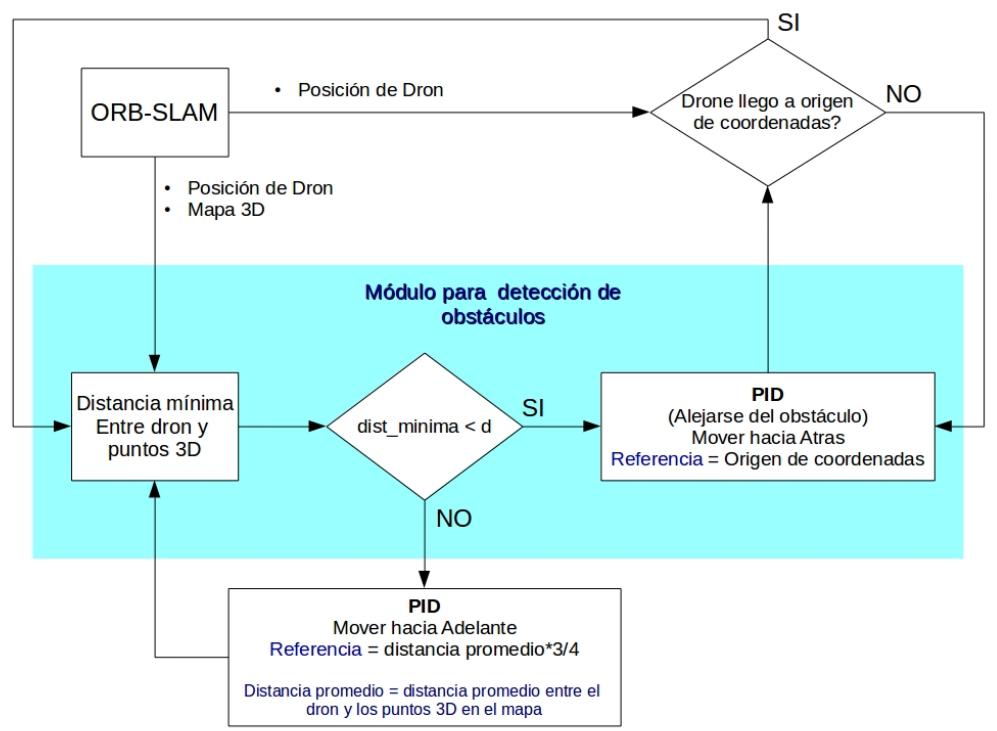

Fig. 4. Diagrama de flujo del esquema de detección de obstáculos propuesto. En el cuadro azul claro se indican los módulos responsables de la detección de un obstáculo cercano al vehículo

\section{Resultados y experimentos}

Para evaluar el funcionamiento de la detección de obstáculos se realizaron dos tipos de experimentos. En el primer experimento se realizó una comparación de

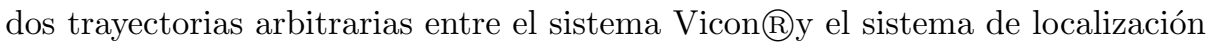
y mapeo ORB-SLAM. En el segundo experimento se evaluó el desempeño de la detección de obstáculos, tomando como sistema de referencia el sistema externo

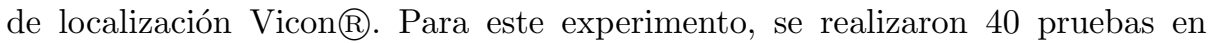
donde el vehículo se desplazó sobre una trayectoria recta y se varió la posición del obstáculo.

Los experimentos se realizaron en un ambiente interior, con una área de trabajo de aproximadamente $6.5 \mathrm{~m}$ X $3 \mathrm{~m}$ (largo X ancho) delimitada virtualmente por el área de cobertura del sistema de seguimiento. Las condiciones de iluminación fueron controladas. La Figura 5 describe la configuración del escenario.

\subsection{Experimento 1: Comparación de sistemas de localización}

En este experimento se comparó la trayectoria obtenida con ORB-SLAM contra el sistema de localización Vicon $\AA$ para determinar la precisión del sistema SLAM. Es importante considerar que el sistema ORB-SLAM es egocéntrico, lo 


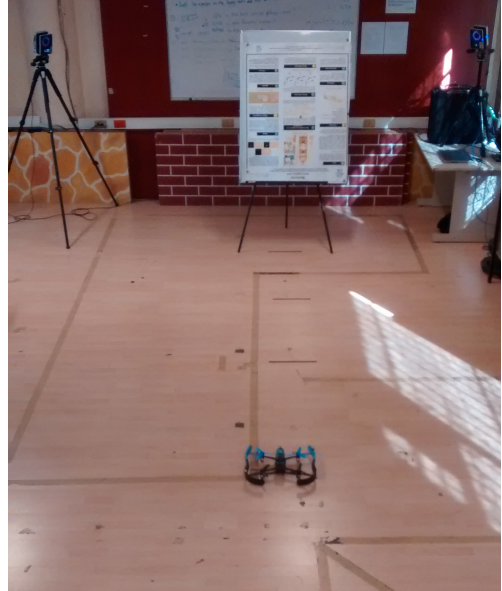

(a) Imagén real del área de trabajo. (b) Representación del área de traEn la imagen se observa el dron en bajo obtenida por el sistema de seel origen del mundo.

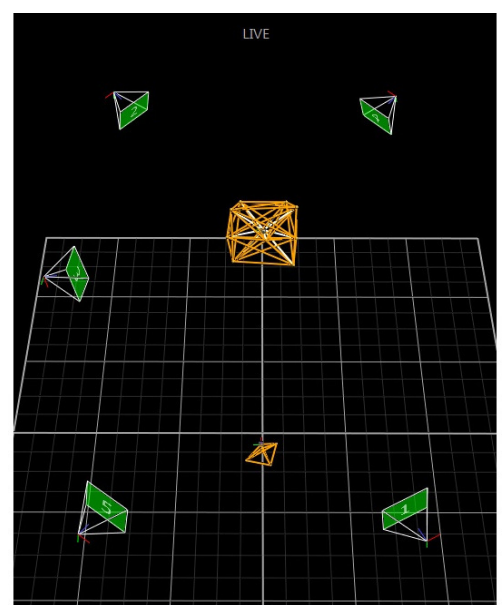
guimiento. En la imagen se observa la distribución de las cámaras (en verde), el dron y el obstáculo.

Fig. 5. Área de trabajo: en ambas imágenes se aprecia el obstáculo en la parte superior y el dron en la parte inferior

cual significa que el origen de referencia se ubica en el punto donde inicia el sistema, por lo tanto, para compara las trayectorias, se realizó una transformación sobre el marco de referencia del sistema ORB-SLAM de tal forma que coinsidiera con el marco de referencia del sistema de seguimiento. Por otro lado, la trayectoria obtenida con el sistema ORB-SLAM tiene una escala arbitraria adimensional, por lo que fue necesario realizar un escalamiento para compararla con el sistema de referencia. ORB-SLAM se ejecutó a una taza aproximada de $30 \mathrm{~Hz}$.

Las pruebas para este primer experimento se realizaron de la siguiente forma:

1. Se despegó el vehículo en cada prueba en el mismo punto de partida.

2. Se inició la captura de posiciones del dron con el sistema de referencia Vicon $\mathrm{R}$.

3. Se inició la captura de posiciones del dron con ORB-SLAM.

4. Se condujo al vehículo a través de una trayectoria aleatoria.

5. Se comparan ambas trayectorias para determinar la precisión de la localización de ORB-SLAM.

En este experimento se realizaron dos pruebas en diferentes configuraciones las cuales están representadas en la Figura 6 para cada prueba se calculó el error cuadrático medio o RMS (Del inglés, Root Mean Square) entre el ORBSLAM y el sistema Viconß. Los resultados detallados se reportan en la Tabla 
1. La trayectoria mostrada en la Figura 6(a) es útil para indicar la habilidad de ORB-SLAM de estimar una trayectoria sin restricción en los grados de libertad (traslación y orientación). Por otra parte, la trayectoria mostrada en la Figura 6(b) es útil para determinar el error en términos porcentuales, puesto que el error es de $5 \mathrm{~cm}$ aproximadamente (ver Tabla 1) sobre una trayectoria de 800 $\mathrm{cm}$, lo cual implica un error porcentual de menos del 1\%. De este modo, este es el error esperado de un sistema SLAM monocular 49] y puede por tanto, ser utilizado con cierto nivel de confianza para estimar la posición del vehículo.

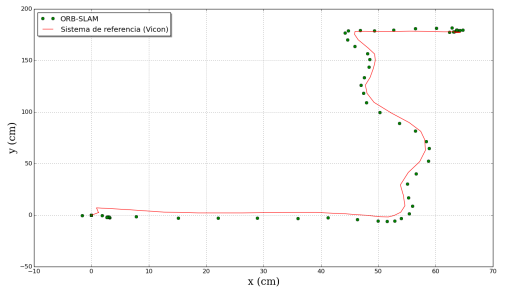

(a) Prueba 1. Trayectoria en forma de L. (b) Prueba 2. Recorrido en forma de rectángulo con cierre de trayectoria.

Fig. 6. Gráficas del Experimento 1. Seguimiento de una trayectoria aleatoria, donde la trayectoria roja representa el seguimiento del Viconß $\cap$ la trayectoria verde representa el seguimiento con el ORB-SLAM

Tabla 1. Resultados del Experimento 1: comparación de sistemas

Resultados del experimento 1
\begin{tabular}{|c|c|}
\hline \# Prueba & RMS $(\mathrm{cm})$ \\
\hline Prueba 1 & 5.2283 \\
\hline Prueba 2 & 5.2794 \\
\hline
\end{tabular}

\subsection{Experimento 2: Detección de obstáculos}

En este experimento se deternimó la capacidad del sistema para la detección de obstáculos. Para evaluar esta detección, el vehículo voló de forma autónoma oscilando entre dos puntos de una linea recta. Durante su recorrido un obstáculo se colocó de frente al vehículo obstruyendo el paso. En este experimento dos elementos fueron rastreados por el sistema Viconßel vehículo y el obstáculo, esto con el fin de calcular la distancia entre ambos elementos. No obstante, es 
importante recalcar que la posición del dron obtenida con ORB-SLAM fue la única información utilizada por el control Proporcional Integral Derivativo que se utilizó para el vuelo autónomo.

Este experimento se realizó con tres configuraciones, donde el obstáculo se colocó a diferentes distancias separadas por 1 metro, como se ve en la Figura 7. Cada configuración está representadas por las letras $a, b, c$, donde $a$ es la configuración más lejana al origen $O$, con una distancia de $2.75 \mathrm{~m}$. Se utilizó un rectángulo sólido de $1.5 \mathrm{~m}$ x $1 \mathrm{~m}$ altamente texturizado como obstáculo. En cada configuración se realizaron 10 pruebas.

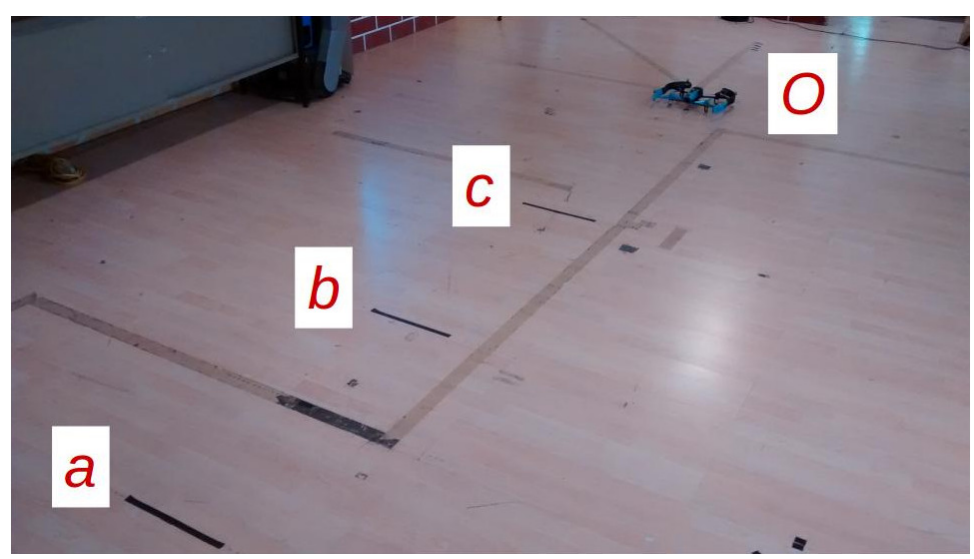

Fig. 7. Configuraciones del Experimento 2. Cada configuración en donde se colocará el obstáculo está representada por las letras $a, b, c$ y $O$ representa el origen del sistema Vicon $\mathrm{R}$

Tabla 2. Tabla de resultados del Experimento 2 para las configuraciones $a$ y $b$, donde d-obs representa la distancia a la que se coloca el obstáculo con respeto al origen del sistema, d-E representa la distancia promedio de detección es decir la distancia mínima entre el vehículo y el obstáculo

\begin{tabular}{|c|c|c|c|c|}
\hline \multicolumn{5}{|c|}{ Resultados del experimento 2 } \\
\hline Configuración & d-obs $(\mathrm{cm})$ & d-E $(\mathrm{cm})$ & \% detección & \% colisión \\
\hline$a$ & 275 & 39.60 & 100 & 0 \\
\hline$b$ & 175 & 45.97 & 80 & 20 \\
\hline
\end{tabular}

Las pruebas se realizaron de la siguiente forma para cada configuración:

1. Se despegó el vehículo en cada prueba en el mismo punto de partida (el origen del sistema Vicon(R). 
Tabla 3. Tabla de resultados del experimento 2 para las configuraciones $c .1$ y $c .2$, donde d-obs representa la distancia a la que se coloca el obstáculo con respecto al origen d-E representa la distancia promedio de detección es decir la distancia mínima entre el vehículo y el obstáculo

\begin{tabular}{|c|c|c|c|c|}
\hline \multicolumn{5}{|c|}{ Resultados del experimento 2 } \\
\hline Configuración & d-obs $(\mathrm{cm})$ & d-E $(\mathrm{cm})$ & \% detección & \% colisión \\
\hline c.1 & 75 & 31.55 & 10 & 90 \\
\hline c.2 & 75 & 64.12 & 100 & 0 \\
\hline
\end{tabular}

2. De forma autónoma el vehículo se movió hasta la pared frontal y retrocedió hasta la pared trasera.

3. Se inició la captura de posiciones del dron con el sistema de referencia

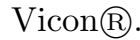

4. Se inició la captura de posiciones del dron con ORB-SLAM.

5. Se inició el vuelo autónomo.

6. Se obstruyó el paso del vehículo cuando viajaba nuevamente hacia la pared frontal.

7. Se calculó distancia mínima a la que se detiene el vehículo antes de colisionar con el obstáculo.

Se realizaron 40 pruebas con las configuraciones antes descritas. Los resultados de cada configuración fueron resumidos en dos tablas. La Tabla 2 resume los resultados obtenidos en las 20 pruebas de las configuraciones $a$ y $b$, donde se utiliza las mismas constantes de control. La Tabla 3 resume 20 pruebas para la configuración $c$ donde, $c .1$ utiliza las mismas constantes de las configuraciones anteriores y $c .2$ emplea constantes reducidas con las que se obtienen mejores resultados. Lo anterior demuestra que el algoritmo de control y detección de obstáculos está sujeto a la sintonización experimental de las constantes del control PID.

La Figura 8(a) ilustra las trayectorias representativas de cada configuración, donde la linea continua representa la trayectoria del dron y la linea punteada representa la trayectoria del obstáculo. Es importante mencionar que en un principio el obstáculo no se interpone en la trayectoria del vehículo, por tanto se aprecia que el dron tiene una trayectoria más prolongada en el principio, sin embargo, cuando el obstáluco es introducido el sistema es capaz de detectarlo y detener al dron para evitar la colisión.

\section{Conclusiones y trabajo futuro}

En este trabajo se ha presentado una metodología para la detección de obstáculos durante vuelo autónomo de un dron. Esta metodología se basa únicamente en el procesamiento de las imágenes obtenidas con una cámara a bordo del dron. Estas imágenes son procesadas por un sistema SLAM monocular que 


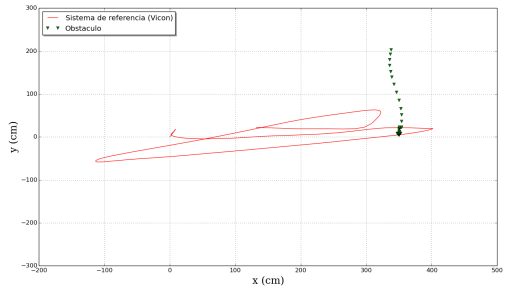

(a) Gráfica representativa de la configura- (b) Gráfica representativa de la configuración $a$.

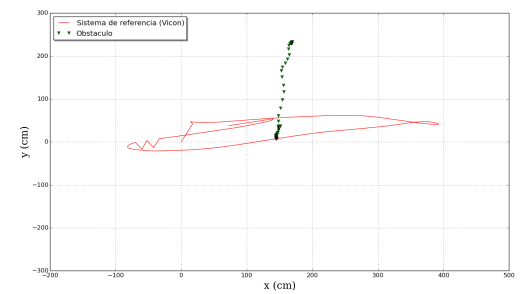

(c) Gráfica representativa de la configura- (d) Gráfica representativa de la configuración c.1.

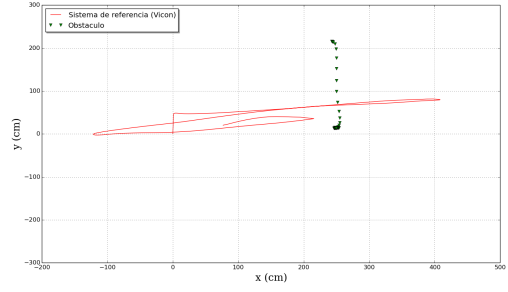
ción $b$.

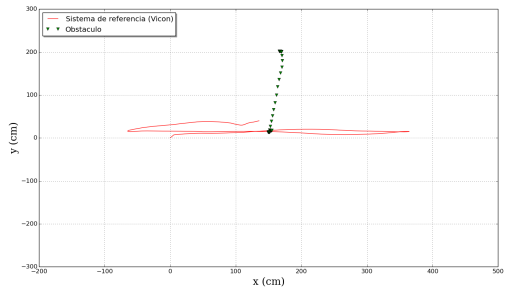

ción $c .2$.

Fig. 8. Gráficas del Experimento 2, donde se dibujan trayectorias representativas de cada configuración

genera una estimación de la posición de dron así como un mapa de puntos en $\mathbb{R}^{3}$ que representan al ambiente, observado con la cámara del dron. De este modo, dicha posición es utilizada por un controlador PID para ejecutar el vuelo autónomo del dron y hacer que éste siga una ruta. Así mismo, el mapa también es utilizado para determinar cuando un objeto, descrito por un conjunto de puntos en $\mathbb{R}^{3}$ en el mapa, aparece en medio de la ruta y por lo tanto, el vuelo debe ser detenido para evitar un impacto contra el objeto.

Adicionalmente, se han presentado resultados que ilustran la efectividad de la metodología propuesta. Con la ayuda de un sistema de localización externo (Vicon), se logró medir la distancia promedio a la que el vehículo logra detenerse a raíz de detectar el obstáculo con un éxito de más del $80 \%$ a una distancia de 40-60 $\mathrm{cm}$ aproximadamente del obstáculo, lo cual permite un frenado suave así como el que el control pueda alejar paulatinamente al dron del obstáculo detectado. Lo anterior indica que el procesamiento de las imágenes se realiza a una tasa suficiente, al menos para el escenario experimental planteado.

Finalmente, este trabajo contribuye a demostrar el tipo de capacidades que pueden ser implementadas para que un dron ejecute un comportamiento inteligente, esto es, el de detectar y así evitar chocar con un obstáculo, utilizando una cámara a bordo como único sensor para observar el ambiente. Lo anterior, es atractivo ya que permite pensar en drones de bajo costo y tamaño reducido, 
equipados con un número reducido de sensores, lo que su vez ahorra energía y peso.

El trabajo a futuro incluye el escalar la metodología presentada en este trabajo a un escenario en exteriores, para rutinas de vuelo más complejas y con obstáculos de diferentes tamaños y texturas.

Agradecimientos. Este trabajo fue financiado por la Royal Society-Newton Advanced Fellowship con referencia NA140454.

\section{Referencias}

1. Albaker, B., Rahim, N.: A survey of collision avoidance approaches for unmanned aerial vehicles. In: technical postgraduates (TECHPOS), 2009 international conference for. pp. 1-7. IEEE (2009)

2. Call, B., Beard, R., Taylor, C., Barber, B.: Obstacle avoidance for unmanned air vehicles using image feature tracking. In: AIAA Guidance, Navigation, and Control Conference. pp. 3406-3414 (2006)

3. Chao, H., Gu, Y., Napolitano, M.: A survey of optical flow techniques for uav navigation applications. In: Unmanned Aircraft Systems (ICUAS), 2013 International Conference on. pp. 710-716. IEEE (2013)

4. Civera, J., Grasa, O.G., Davison, A.J., Montiel, J.M.M.: 1-point ransac for ekfbased structure from motion. In: International Conference on Intelligent Robots and Systems. pp. 3498-3504. IEEE (2009)

5. Ding, W., Wang, J., Han, S., Almagbile, A., Garratt, M.A., Lambert, A., Wang, J.J.: Adding optical flow into the gps/ins integration for uav navigation. In: Proc. of International Global Navigation Satellite Systems Society Symposium. pp. 1-13. Citeseer (2009)

6. Engels, C., Stewénius, H., Nistér, D.: Bundle adjustment rules. Photogrammetric computer vision 2, 124-131 (2006)

7. Griffiths, S., Saunders, J., Curtis, A., Barber, B., McLain, T., Beard, R.: Obstacle and terrain avoidance for miniature aerial vehicles. In: Advances in Unmanned Aerial Vehicles, pp. 213-244. Springer (2007)

8. Hrabar, S.: 3d path planning and stereo-based obstacle avoidance for rotorcraft uavs. In: Intelligent Robots and Systems, 2008. IROS 2008. IEEE/RSJ International Conference on. pp. 807-814. IEEE (2008)

9. Martinez-Carranza, J., Calway, A.: Efficient ekf-based visual odometry using a structure-driven temporal map. In: International Conference on Robotics and Automation ICRA. IEEE (May 2012)

10. Martınez-Carranza, J., Loewen, N., Márquez, F., Garcıa, E.O., Mayol-Cuevas, W.: Towards autonomous flight of micro aerial vehicles using orb-slam. In: IEEE 3rd Workshop on Research, Education and Development of Unmanned Aerial Systems, RED-UAS (2015)

11. Mueller, T.J.: Fixed and flapping wing aerodynamics for micro air vehicle applications, vol. 195. AIAA (2001)

12. Mur-Artal, R., Montiel, J., Tardos, J.D.: Orb-slam: a versatile and accurate monocular slam system. Robotics, IEEE Transactions on 31(5), 1147-1163 (2015)

13. Roberts, J.M., Corke, P.I., Buskey, G.: Low-cost flight control system for a small autonomous helicopter. In: Australasian Conference on Robotics and Automation. Auckland, New Zealand (2002) 
14. Scherer, S., Singh, S., Chamberlain, L., Saripalli, S.: Flying fast and low among obstacles. In: Robotics and Automation, 2007 IEEE International Conference on. pp. 2023-2029. IEEE (2007)

15. Shim, D., Chung, H., Kim, H.J., Sastry, S.: Autonomous exploration in unknown urban environments for unmanned aerial vehicles. In: Proc. AIAA GN\&C Conference $(2005)$

16. Silveira Vidal, F., de Oliveira Palmerim Barcelos, A., Ferreira Rosa, P.F.: Slam solution based on particle filter with outliers filtering in dynamic environments. In: Industrial Electronics (ISIE), 2015 IEEE 24th International Symposium on. pp. 644-649. IEEE (2015)

17. Watanabe, Y., Calise, A.J., Johnson, E.N.: Vision-based obstacle avoidance for uavs. In: AIAA Guidance, Navigation and Control Conference and Exhibit. pp. 20-23 (2007)

18. Zhou, G., Fang, L., Tang, K., Zhang, H., Wang, K., Yang, K.: Guidance: A visual sensing platform for robotic applications. In: Proceedings of the IEEE Conference on Computer Vision and Pattern Recognition Workshops. pp. 9-14 (2015)

19. Zufferey, J.C., Floreano, D.: Toward 30-gram autonomous indoor aircraft: Visionbased obstacle avoidance and altitude control. In: Robotics and Automation, 2005. ICRA 2005. Proceedings of the 2005 IEEE International Conference on. pp. 25942599. IEEE (2005) 